\title{
Focused Image creation approaches for macroscopic objetcs encoded in digital holograms
}

\author{
$\underline{\text { C. P. Mc Elhinney }}^{a}$, B. M. Hennelly ${ }^{a}$ and T. J. Naughton ${ }^{a, b}$ \\ ${ }^{a}$ Dept. of Computer Science, National University of Ireland Maynooth, Co. Kildare, Ireland \\ ${ }^{b}$ University of Oulu, RFMedia Laboratory, Oulu Southern Institute, Vierimaantie 5, 84100 \\ Ylivieska, Finland
}

\begin{abstract}
When a digital hologram is reconstructed only points on objects within the depth of focus at the reconstruction distance are in focus. For complex scenes, scenes containing multiple objects or multiple object features located at different depths, this can lead to a reconstruction with large blurred regions. Using a depth-from-focus algorithm we have developed an approach to extract an objects depth information in the form of a depth map from volumes of reconstructions, where each reconstruction in the volume is a reconstruction at a different focal plane. By combining the depth map with the volume of reconstructions used to calculate the depth map we can create an image, an extended focus image, where the full scene is in focus. To our knowledge, this is the first technique which creates extended focused images of digital holograms encoding macroscopic objects. We present results for digital holograms containing low and high contrast macroscopic objects.
\end{abstract}

Keywords: digital holgraphy, image processing, three-dimensional image processing, focus detection, depth estimation, focused imaging

\section{INTRODUCTION}

Holography is an established technique for recording and reconstructing real-world three-dimensional (3D) objects. Digital holography ${ }^{1-4}$ and digital holographic image processing ${ }^{5-7}$ have recently become feasible due to advances in megapixel CCD sensors with high spatial resolution and high dynamic range. In the case of recording, the most commonly used techniques include in-line phase-shifting interferometry (PSI) and off-axis digital holography ${ }^{1,3,8}$ which allow for the removal of the unwanted intensities and virtual image that are inherent in holography. A single-exposure on-line (SEOL) set-up ${ }^{9-11}$ is also possible due to the availability of digital techniques to remove the unwanted terms. ${ }^{1}$ In the case of reconstruction, algorithms are applied to the recorded interferogram/holograms in order to view the object under investigation. Numerous techniques exist based on discrete implementations of the Fresnel transform. ${ }^{3}$ In the work presented here we employ PSI to capture our in-line digital holograms (DHs), which are in an appropriate form for data transmission and digital image processing.

Digital holographic image processing is a field which has seen increased interest in recent few years. The large file sizes of DHs has lead to research into their compression for the purpose of efficient storage and transmission. ${ }^{12}$ The research has focused on compression of the complex field at the hologram plane $e^{5,12-15}$ and the reconstruction plane. ${ }^{16}$ Digital holographic reconstructions also suffer from speckle noise, a noise that is inherent in any optical system using coherent light. The digital nature of DHs allows postprocessing of reconstructions to reduce speckle noise and improve image quality. ${ }^{17,18}$ Through the use of incoherent object illumination, optical scanning holography produces speckle-free reconstructions. ${ }^{19}$ Reconstructions can also be improved through processing of the recorded hologram to remove or suppress error sources such as the twin-image ${ }^{20}$ and the dc-term. ${ }^{1,3}$ These terms can also be filtered from the hologram optically. ${ }^{21,22}$ These properties along with the unique properties of DHs (such as the application of phase-contrast technologies and reconstruction at an arbitrary focal plane) have led to increased research in the field of digital holographic microscopy. ${ }^{1,23-26}$ The recording of biological data using digital holographic microscopy has numerous advantages over optical microscopy such

Further author information: CMc: conormce@cs.nuim.ie; TN: tomn@cs.nuim.ie

Photon Management III, edited by John T. Sheridan, Frank Wyrowski

Proc. of SPIE Vol. 6994, 69940U, (2008) · 0277-786X/08/\$18 - doi: 10.1117/12.782781 
as shape recovery, ${ }^{27,28}$ real-time tracking of the focal plane ${ }^{26}$ and the creation of images where the full sample is in focus. ${ }^{29}$ This form of microscopy has also renewed interest in the fields of digital holographic object segmentation ${ }^{30-32}$ and recognition..$^{9,33-35}$

A disadvantage of holographic reconstructions is the limited depth of field. When a digital hologram is reconstructed, a distance value $d$ is input as a parameter to the reconstruction algorithm. The depth of focus range for a reconstruction using the Fresnel approximation is in the order of a millimeter. For complex 3D scenes, scenes containing multiple objects or containing multiple object features located at different depths, this leads to reconstructions with large blurred regions. We are interested in the creation of an image with an extended depth of field, ${ }^{36}$ which we are calling an extended focus image (EFI) ${ }^{29}$ from sets of digital holographic reconstructions where the full scene is in focus. To do this we must be able to identify when certain object regions are in focus at a given depth. This effectively necessitates the development of a shape extraction technique for macroscopic objects encoded in digital holograms. ${ }^{32,37}$ An EFI technique has been previously developed for microscopic objects recorded using digital holography, ${ }^{29}$ but the shape extraction technique they employ is not applicable to our macroscopic objects primarily due to the corruptive effect of speckle noise which is not present in $\mathrm{DH}$ microscopy.

In this paper we present a method for creating EFIs from sets of digital holographic reconstructions of macroscopic objects. We first create a depth map using a depth-from-focus (DFF) technique. This technique recovers depth information by calculating a focus measure on blocks of multiple reconstructions of the $\mathrm{DH}$. We then combine the depth map and the reconstructions to create an EFI. We discuss a method for creating an EFI in a short length of time using a non-overlapping block approach and a method for creating a qualitatively more accurate EFI using an overlapping block approach. We also demonstrate EFIs created on both low and high-contrast scenes, and discuss the advantages and disadvantages of the technique. Section. 2 discusses the recording process for PSI DHs and our experimental setup. In Sect. 3 we introduce focus and focus detection for DHs. The algorithms for calculating a depth map using an overlapping and non-overlapping DFF approach are discussed in detail. Section. 4 presents a sequential discussion of our different EFI creation approaches along with their advantages and disadvantages. This section provides experimental results of EFIs created from DHs encoding real-world 3D objects. In Sect. 5 some conclusions are drawn about the benefits and limitations of the different EFI creation algorithms.

\section{PHASE-SHIFT DIGITAL HOLOGRAPHY}

We record interferograms with an optical system based on a Mach-Zehnder interferometer (see Fig. 1). The interferograms are real-valued images resulting from the interference between an object wave and a reference wave. In our system, a linearly polarized Helium-Neon $(632.8 \mathrm{~nm})$ laser beam is expanded and collimated, and split into object and reference waves. The object wave illuminates an object placed at a distance $d$ that is selected based on the object size in order to avoid aliasing of the CCD. ${ }^{38}$ Our CCD camera has $2048 \times 2032$ pixels of size $7.4 \mu \mathrm{m}$ in both dimensions. We denote $U_{0}(x, y)$ as the complex amplitude distribution immediately in front of the $3 \mathrm{D}$ object. The reference wave passes through $\mathrm{RP}_{1}$ and $\mathrm{RP}_{2}$, and by selectively rotating the plates we can achieve four phase shift permutations. An interferogram is recorded for every phase shift and we then use these four interferograms and a four frame $\mathrm{PSI}^{4,39}$ algorithm to compute the camera plane complex field $H_{0}(x, y)$, the DH. PSI is a digital holographic technique that calculates in-line holograms free of the twin-image and dc-term.

We numerically reconstruct a DH in a plane at any distance $z$ from the camera using the Fresnel transform..$^{2,4,33}$ This is achievable because a DH, $H_{0}(x, y)$, contains sufficient amplitude and phase information to reconstruct the complex field $U_{z}(x, y)$. This can be calculated from the Fresnel approximation ${ }^{40}$ as

$$
U_{z}(x, y)=\frac{-\mathrm{i}}{\lambda z} \exp \left(\mathrm{i} \frac{2 \pi}{\lambda} z\right) H_{0}(x, y) \star \exp \left[\mathrm{i} \pi \frac{\left(x^{2}+y^{2}\right)}{\lambda z}\right],
$$

where $\lambda$ is the wavelength of the light and $\star$ denotes a convolution. At $z=d$, and ignoring errors in digital propagation due to pixelation and rounding, the discrete reconstruction $U_{Z}(x, y)$ is a close approximation of the physical continuous field $U_{0}(x, y)$. As with conventional holography, a $\mathrm{DH}$ encodes different views of a $3 \mathrm{D}$ object from a small range of angles. ${ }^{40,41}$ However, we only demonstrate the creation of EFIs using an individual perspective. In the next section we introduce focus and how the relative focus of images can be be calculated.

Proc. of SPIE Vol. 6994 69940U-2 


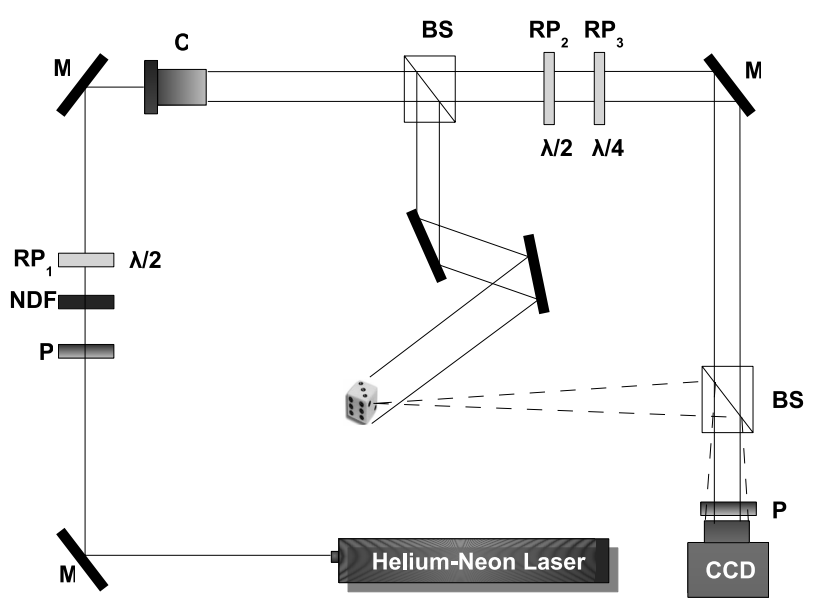

Figure 1. Experimental setup for PSI: P, polarizer; NDF, neutral density filter; C, collimator; BE, beam expander; BS, beam splitter; RP, retardation plate; $\mathrm{M}$, mirror.

\section{FOCUS DETECTION IN DIGITAL HOLOGRAMS}

All imaging systems generally have a finite depth of field. The recorded image can either be in focus or out of focus. The objects which lie within the depth of field of the imaging system are in focus (appearing sharp) while the objects which lie outside of the depth of field of the system are out of focus (appearing blurred). While the development and application of focus measures in incoherent imaging has been well studied, there exists no definitive criterion for finding the focal plane of a scene or finding the focal distance for a region within a scene. In the field of digital holography, the study of focus measures has not received the same interest. However, focus techniques have been applied to both reconstructions of digitally recorded holograms ${ }^{26,29,32,42-44}$ and to the digitized reconstructions of optically recorded holograms. ${ }^{37,45,46}$ These employ focus measures such as selfentropy, ${ }^{42}$ phase changes, ${ }^{26}$ wavelet analysis, ${ }^{43}$ gray level variance, ${ }^{37}$ and integrated amplitude modulus ${ }^{44}$ among others. Using these measures, applications such as the detection of the focal plane ${ }^{26,43,44}$ in digital holographic microscopy, the measurement of 3D objects in the digitized reconstructions of physical holograms, ${ }^{?, 37}$ the segmentation of macroscopic objects ${ }^{32}$ and the creation of EFIs for microscopic objects ${ }^{29}$ have been demonstrated. We now proceed to focus detection and demonstrate how using a focus measure we can estimate the depth of pixel blocks in digital holographic reconstructions.

\subsection{Focus detection}

To determine if an image is in-focus the accepted procedure is to record a set of images with either a dynamic scene or to record a set of images with a static scene where the focal plane of the camera is varied. A function is then applied to each image and the image which maximizes this function is taken as the in-focus image. Focus measures are also known as sharpness functions originating from the work of Muller et al. ${ }^{\text {? }}$ where they were the first to apply a function to an image to determine focus, which they called an 'image sharpness function'. Modern focus measures are based on the assumption that edges are more defined in focused images and are therefore more sharp (have higher spatial frequencies). Numerous sharpness based focus measures have been developed and evaluated ${ }^{47}$ for incoherent imaging which generally satisfy the following requirements:

- independent of image content

- low computation complexity

- focus measure should be unimodal

- there should be a large variation in returned value with respect to blurring

- robust to noise

Proc. of SPIE Vol. $699469940 U-3$ 


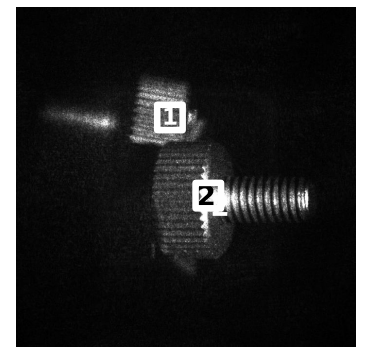

(a)

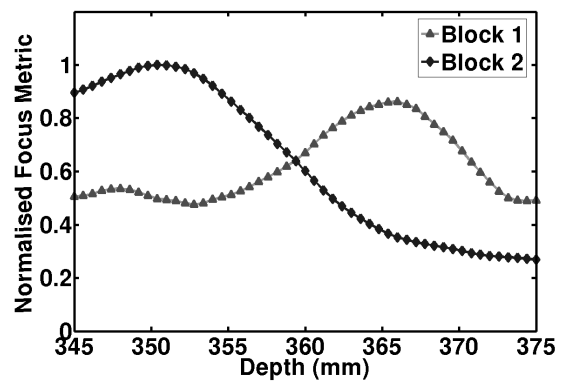

(b)

Figure 2. Two bolts object DH: (a) numerical reconstruction, (b) variance plot for object blocks 1 and 2 .

Focus measures are functions which attempt to determine the relative level of focus of sets of images. The accepted image property maximized by these functions is the high spatial frequency energy of the image. ${ }^{47}$ Variance is a focus measure calculated on the intensity of an image using

$$
V\left(I_{z}\right)=\frac{1}{n^{2}} \sum_{i=1}^{n} \sum_{j=1}^{n}\left[I_{z}(i, j)-\bar{I}_{z}\right]^{2}
$$

where $I_{z}$ is an image or image region, of size $n \times n$, indexed by pixel locations $i$ and $j$ and where $\overline{I_{z}}$ is the arithmetic mean of $I_{z} . V\left(I_{z}\right)$ is therefore a focus value for the image, or image region, $I_{z}$. Variance has been proven to be a sound focus measure. ${ }^{47}$ An example of the application of variance as a focus measure is given in Fig. 2. We selected one $81 \times 81$ block of pixels on each of the two bolts in the DH. A reconstruction of the DH with the two blocks highlighted is shown in Fig. 2(a). The variance on the object blocks is calculated from 151 reconstructions spanning the focal range of the objects in the DH. The focal depth for each object block is selected by choosing the reconstruction depth which returns the highest variance value for that block. The variance plots for the objects blocks are shown in Fig. 2(b). The returned focal depths for the object blocks, obtained by selecting the depth which quantitatively has the maximum focus value, correspond to the depths we obtained by visually selecting the most in focus depths.

\subsection{Depth-from-focus}

DFF is an image processing approach for the estimation of surface shape in a scene using multiple independently focused images. DFF approaches estimate the focal plane of a DH by maximizing a focus measure, such as equation. 2, which is applied to the intensity of several 2D reconstructions, where each reconstruction is at a different focal plane. Ma et al. ${ }^{37}$ first proposed a DFF algorithm applied to digitized physical holograms for the recovery of 3D shape information from digitized physical holograms. By calculating variance on non-overlapping blocks from reconstructions of a DH at different depths they recovered depth information from a lower-resolution version of the sensed object. We choose to extend this variance-measurement approach in order to classify each $1 \mathrm{D}$ vector $(x, y)$ in the reconstruction volume (each line of pixels parallel to the optical axis) as either belonging to the object or belonging to the background. ${ }^{32}$ The decision is taken as follows: if vector $(x, y)$ contains an in focus pixel from the object at any depth $z$ then $(x, y)$ is an object pixel, otherwise it is a background pixel.

Each reconstruction $I_{z}(k, l)=\left|U_{z}(k, l)\right|^{2}$ is of size $M \times N$ pixels. Our algorithm requires five input parameters: a DH, a block size $n \times n$, a start depth $z_{\min }$, an increment $z_{\text {step }}$ and an end depth $z_{\text {max }}$. The initial reconstruction depth $z$ is set to the starting depth, $z=z_{\min }$. The algorithm involves the following three steps as illustrated in Fig. 3:

Step 1: The input DH is reconstructed at depth $z$ and a speckle reduction technique can be applied. Speckle reduction is an optional part of the process where the speckle reduction technique to be used is chosen by the user. The output reconstructions intensity is stored in $I_{z}(k, l)$.

Step 2: We then calculate variance for each pixel by calculating variance on $n \times n$ pixel overlapping blocks 


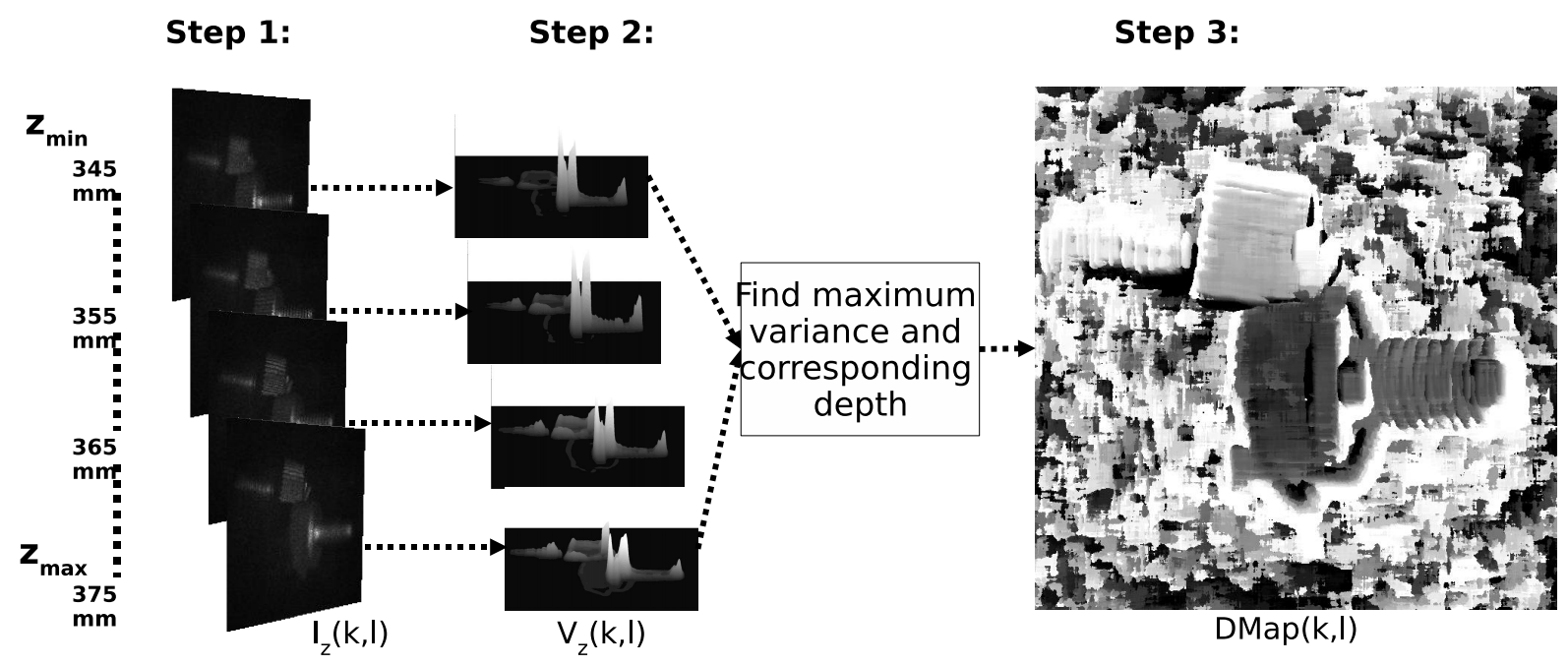

Figure 3. Depth-from-focus process, Step 1: numerically reconstruct over a range of depths, Step 2: block process each reconstruction using variance, Step 3: calculate depth map.

approximately centered on each pixel, and address each block with $(k, l)$ where $k \in[0,(M-1)], l \in[0,(N-1)]$. Variance of each overlapping block at each depth $z$ is calculated with function $V_{z}: \mathbb{R}^{n \times n} \rightarrow \mathbb{R}^{+}$defined by

$$
V_{z}(k, l)=\frac{1}{n^{2}} \sum_{x=k-\left\lfloor\frac{n-1}{2}\right\rfloor}^{k+\left\lceil\frac{n-1}{2}\right\rceil} \sum_{y=l-\left\lfloor\frac{n-1}{2}\right\rfloor}^{l+\left\lceil\frac{n-1}{2}\right\rceil}\left[I_{z}(x, y)-\overline{I_{z}(k, l)}\right]^{2},
$$

$V$ is therefore a volume storing a $2 \mathrm{D}$ variance image for each depth $z$ and $I_{z}(k, l)$ is defined as

$$
\overline{I_{z}(k, l)}=\frac{1}{n^{2}} \sum_{x=k-\left\lfloor\frac{n-1}{2}\right\rfloor}^{k+\left\lceil\frac{n-1}{2}\right\rceil} \sum_{y=l-\left\lfloor\frac{n-1}{2}\right\rfloor}^{l+\left\lceil\frac{n-1}{2}\right\rceil} I_{z}(x, y) .
$$

For the non-overlapping algorithm the volume is calculated with

$$
V_{z}^{\mathrm{no}}(r, s)=\frac{1}{n^{2}} \sum_{x=r n}^{r n+n-1} \sum_{y=s n}^{s n+n-1}\left[I_{z}(x, y)-\overline{I_{z}\left(r n+\left\lfloor\frac{n-1}{2}\right\rfloor, s n+\left\lfloor\frac{n-1}{2}\right\rfloor\right)}\right]
$$

where $r \in[0,1, \ldots\lfloor M / n\rfloor]-1, s \in[0,1, \ldots\lfloor N-/ n\rfloor-1]$.

Step 3: The next step is to calculate the depth map. In the overlapping case, for each pixel $(k, l)$ we find the maximum value in $\mathrm{V}_{z}(k, l)$. The depth where this maximum occurs, $z$, is then stored in DMap $(k, l)$. The process is the same for the non-overlapping case except we find the maximum value in $\mathrm{V}_{z}^{\mathrm{NO}}(r, s)$ and store the depth in $\operatorname{DMap}(r, s)$. This algorithm has been extended to create a maximum variance map which can be employed to segment object from background in a DHs reconstruction. ${ }^{32}$ We are now in a position to create EFIs by combining $\operatorname{DMap}(k, l)$ with the volume of reconstructions $I_{z}(k, l)$.

\section{EXTENDED FOCUSED IMAGING}

In all the experiments in this paper, we use a block size of $81 \times 81$ as input to the DFF algorithm. Larger block sizes have the advantage of estimating the general shape of an object with low error but the shape of finer object features is lost. Conversely, the smaller block sizes have the advantage of estimating the shape of finer object features but at the cost of high error in the estimate of the general shape of the object. We use the recommended block size of $81 \times 81$ for DHs containing macroscopic objects. ${ }^{32}$ We apply the digital speckle reduction technique of discrete Fourier filtering developed by Maycock et al. ${ }^{18}$ to all our reconstructions and selected this technique 

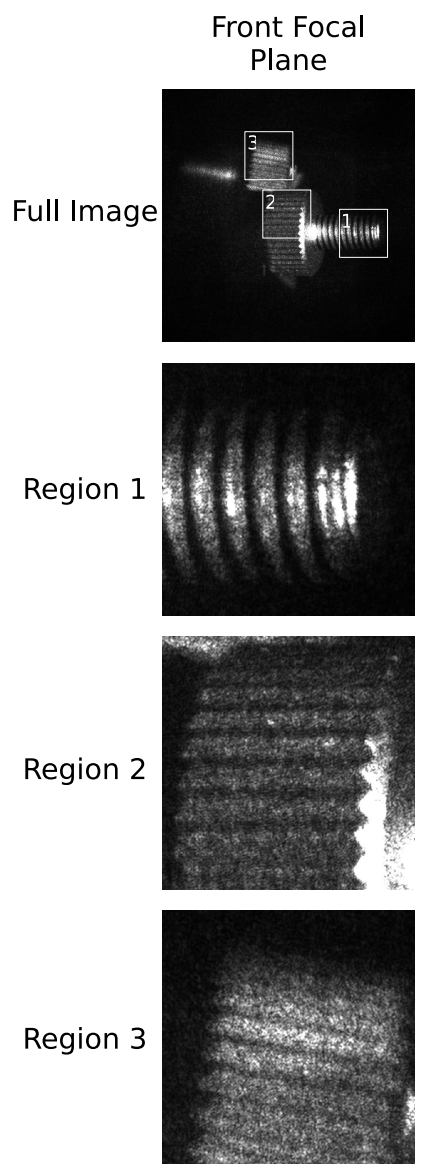
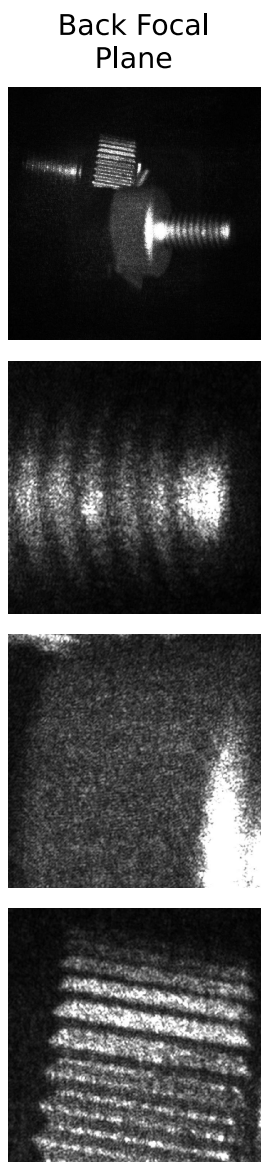

EFI- Non-Overlapping 11 Reconstructions
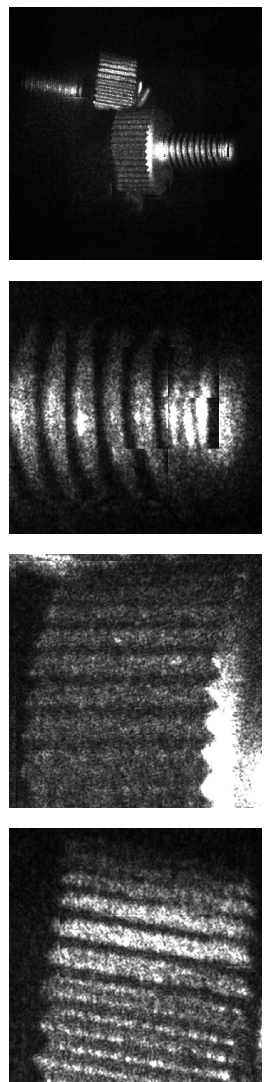

EFI- Non-Overlapping 151 Reconstructions
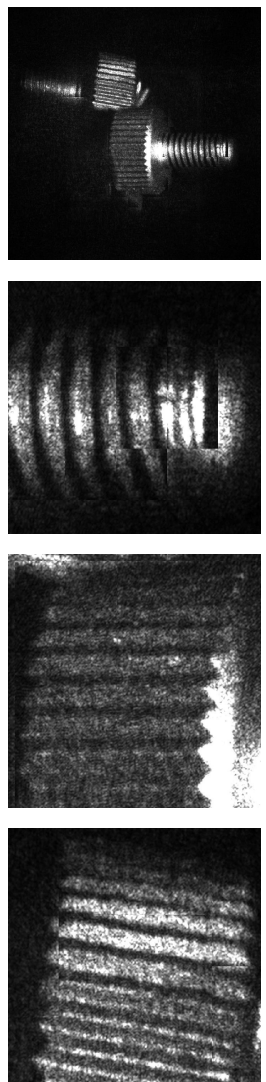

Figure 4. Two bolts object DH, reconstructions and the non-overlapping approach EFIs.

as it has been shown to provide good results for DHs containing macroscopic objects. This speckle reduction technique is based on applying different non-overlapping band pass filters to a complex reconstruction and summing the intensities of the resultant complex images. The depth of focus of the reconstructions used in these experiments ranges from a minimum of $0.4 \mathrm{~mm}$ to a maximum of $0.8 \mathrm{~mm} .^{1}$ In this section we will demonstrate EFIs with a depth of focus ranging from $6 \mathrm{~mm}$ to $20 \mathrm{~mm}$.

\subsection{Non-Overlapping}

By using the non-overlapping DFF algorithm, low resolution depth maps can be created. Through combining a depth map with numerical reconstructions of the $\mathrm{DH}$ we can create EFIs. For each pixel in $\operatorname{DMap}(r, s)$, an $n \times n$ pixel block of intensity values is mapped to the non-overlapping-case EFI as

$$
\operatorname{EFI}_{\mathrm{NO}}(k, l)=I_{\mathrm{DMap}(r, s)}(k, l),
$$

where $r=\lfloor k / n\rfloor, s=\lfloor l / n\rfloor$, where the notation $I_{\mathrm{DMap}(r, s)}(k, l)$ denotes the real-valued intensity value at lateral coordinates $(k, l)$ and depth $\operatorname{DMap}(r, s)$ in the reconstruction volume, where $k \in\left[0,1, \ldots, M^{\prime}\right], l \in\left[0,1, \ldots, N^{\prime}\right]$, and where $M^{\prime}=n\lfloor M / n\rfloor, N^{\prime}=n\lfloor N / n\rfloor$ (for the nonoverlapping case only, the $M^{\prime} \times N^{\prime}$ pixel dimensions of the EFI will be smaller than the the original reconstructions if $n$ does not divide $M, N)$.

In Fig. 4 two numerical reconstructions, from the front focal plane and back focal plane of the DH, are displayed alongside the $\mathrm{EFI}_{\mathrm{NO}}(k, l)$ reconstructions. The objects in this DH have a depth of focus of approximately $20 \mathrm{~mm}$. We created two $\mathrm{EFI}_{\mathrm{NO}}(k, l)$ s using 11 numerical reconstructions and 151 reconstructions, where using a greater number of reconstructions results in a more accurate depth map and therefore a more accurate EFI. To compare these two $\operatorname{EFI}_{\mathrm{NO}}(k, l)$ reconstructions we selected three object regions which are labelled in Fig. 4: two 


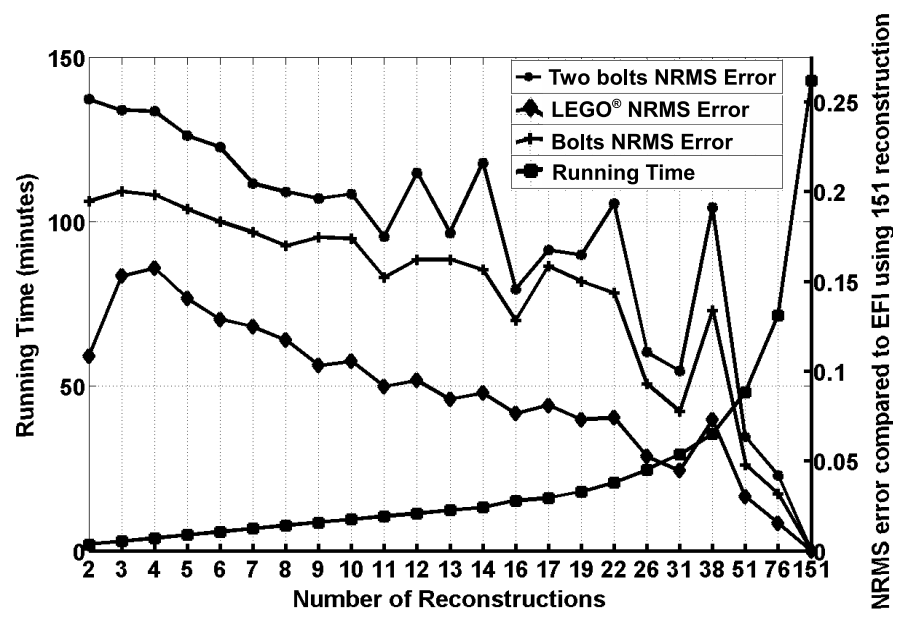

Figure 5. Accuracy and timing plot for the non-overlapping approach EFIs created using increasing numbers of reconstructions.

on the front screw object and one on the back screw object. The numerical reconstructions in Fig. 4 illustrate the limited depth of field of a DH reconstruction, while the output $\operatorname{EFI}_{\mathrm{NO}}(k, l)$ demonstrate how our technique can overcome this limitation to create images where all objects are in focus.

Due to the large size of DHs, $2048 \times 2048$, the DFF algorithm, and consequently the EFI creation, is computationally intensive. On a $\mathrm{P} 43 \mathrm{GHz} \mathrm{PC}$ the creation of the $\operatorname{EFI}_{\mathrm{NO}}(k, l)$ using 151 reconstructions takes almost 2.5 hours. We investigated the impact of reducing the number of reconstruction on the output EFI for all the DHs presented in this paper. By taking the $\operatorname{EFI}_{\mathrm{NO}}(k, l)$ created using a large number of reconstructions (151) as our best EFI we compared the $\operatorname{EFI}_{\mathrm{NO}}(k, l)$ created using different numbers of reconstructions on two criteria: running time and normalized root-mean-square error (NRMS) error. ${ }^{48}$ Plots of running time and NRMS error as a function of the number of reconstructions used in the $\operatorname{EFI}_{\mathrm{NO}}(k, l)$ creation are shown in Fig 5 . The jumps in error value in Fig. 5 occur when our sampling of the reconstruction space does not include the depth for some of the large object regions. This leads to a depth with incorrect estimates of depths in object regions, causing blurring in the resulting $\operatorname{EFI}_{\mathrm{NO}}(k, l)$ and an increase in error. For some scenes, it can happen that a lower sampling of depths will include more exact depths at which object regions are located. In a short period of time, less than ten minutes, an $\operatorname{EFI}_{\mathrm{NO}}(k, l)$ using only eleven reconstructions can be created with an error of $17.5 \%$ when compared to our qualitative best EFI. A visual comparison of the two non-overlapping EFIs is displayed in Fig. 4. Both of the $\mathrm{EFI}_{\mathrm{NO}}(k, l)$ reconstructions produce images of the scene where both objects are in focus. They also both struggle to select the correct blocks for the tip of the front screw as shown in the row of region 1 images in Fig. 4. These EFIs can be improved on through the use of the overlapping DFF algorithm to create higher resolution depth maps prior to the calculation of an EFI.

\subsection{Overlapping}

We have developed an approach for creating EFIs from depth maps calculated using the overlapping DFF algorithm. The pointwise approach produces a sharp EFI but does not attempt to compensate for any errors in $\operatorname{DMap}(k, l)$ which can occur for a number of reasons including poor object illumination or speckle noise. A neighborhood approach ${ }^{49}$ has been developed which applies smoothing based on the $n \times n$ block size used to create $\operatorname{DMap}(k, l)$. The overlapping algorithm takes, on average, 25 minutes to process an individual reconstruction compared to less than one minute for the non-overlapping algorithm. However, The overlapping approach returns a high resolution $\operatorname{DMap}(k, l)$ allowing for a higher quality EFI.

\subsubsection{Pointwise approach}

To calculate the pointwise EFI, $\operatorname{EFI}_{\mathrm{P}}(k, l)$, we take the depth for each pixel from $\operatorname{DMap}(k, l)$ and store the intensity value of the corresponding pixel from $I_{z}(k, l)$ for that depth in $\operatorname{EFI}_{\mathrm{P}}(k, l)$. We calculate $\operatorname{EFI}_{\mathrm{P}}(k, l)$ with the following function

$$
\operatorname{EFI}_{\mathrm{P}}(k, l)=I_{\mathrm{DMap}(k, l)}(k, l)
$$




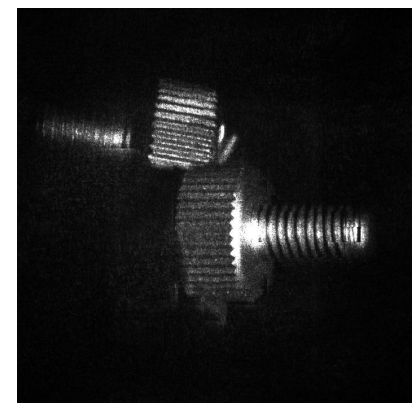

(a)

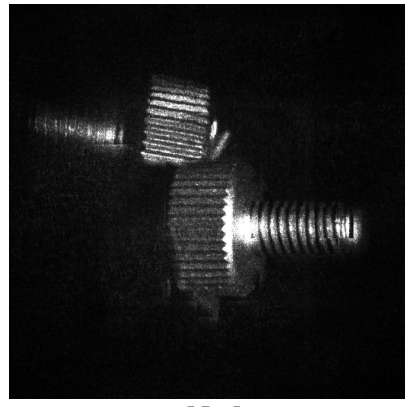

(b)

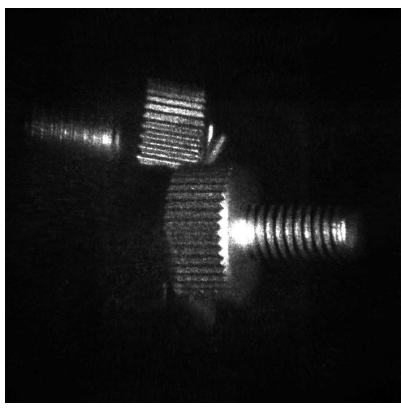

(c)

Figure 6. Two bolts object EFIs created using the (a) non-overlapping approach and 11 reconstructions, (b) nonoverlapping approach and 151 reconstructions and (c) overlapping pointwise approach and 151 reconstructions.

\section{Front Focal}

Plane

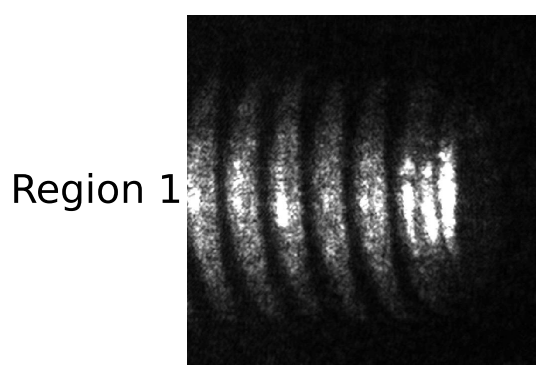

Back Focal

Plane

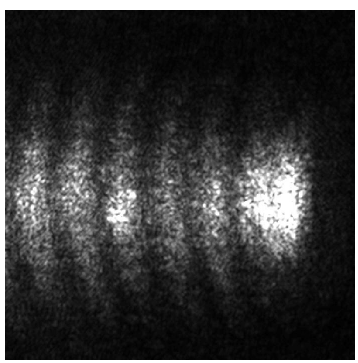

EFI- Pointwise

Approach

Figure 7. Two bolts object DH reconstructions for region 1 and the overlapping approach EFIs.

where $k \in[0,(M-1)], l \in[0,(N-1)]$.

A comparison of the two overlapping approach, $\operatorname{EFI}_{\mathrm{P}}(k, l)$, and the two $\operatorname{EFI}_{\mathrm{NO}}(k, l)$ reconstructions, detailed in Sect. 4.1, is displayed in Fig. 6. The overlapping approach produces a more accurate EFI as can be seen by comparing, in particular, object region 1 from the $\operatorname{EFI}_{\mathrm{NO}}(k, l)$, see Fig. 4, with object region 1 from the calculated $\operatorname{EFI}_{\mathrm{P}}(k, l)$, see Fig. 7. In some cases the depth of object regions may be incorrectly estimated leading to errors in $\operatorname{EFI}_{\mathrm{P}}(k, l)$. This is apparent in Fig. 7 where there are still some errors in the top of the threads of the screw. However, by sacrificing some image sharpness a neighborhood approach ${ }^{49}$ as been developed which reduces the error in the output EFI.

To illustrate our techniques effectiveness on low contrast objects we calculated $\operatorname{EFI}_{\mathrm{P}}(k, l)$ for a $\operatorname{LEGO}{ }^{\circledR}$ block object DH, with a depth of focus of $6 \mathrm{~mm}$. In Fig. 8 a front focal plane and back focal plane reconstruction are shown alongside $\operatorname{EFI}_{\mathrm{P}}(k, l)$. We identified two object regions: the back LEGO ${ }^{\circledR}$ block, region 1, and the front LEGO ${ }^{\circledR}$ block, region 2, both with the word "LEGO ${ }^{\circledR}$ " inscribed. In these reconstructions the word "LEGO ${ }^{\circledR} "$ is only legible in one of the reconstructions, region 2 and region 1 in the front focal and back focal plane respectively. However, in $\operatorname{EFI}_{\mathrm{P}}(k, l)$ the word "LEGO ${ }^{\circledR}$ " is legible in both region 1 and region 2. Figure 9 and Figure 10 contain a front focal plane reconstruction, a back focal plane reconstruction and $\operatorname{EFI}_{\mathrm{P}}(k, l)$ for two DH containing: one containing two different types of bolts and one containing a large bolt Again is it clear that the EFI contains all in focus regions from both of these images. These EFIs of DHs containing different objects and objects of varying levels of contrast demonstrate the effectiveness of our approach to create focused images out of a volume of DHs reconstructions.

\section{CONCLUSION}

The field of focus detection and its recent application to digital holographic reconstructions has been discussed in this paper. We have detailed a novel method for creating an image where all objects are in focus, an EFI, 

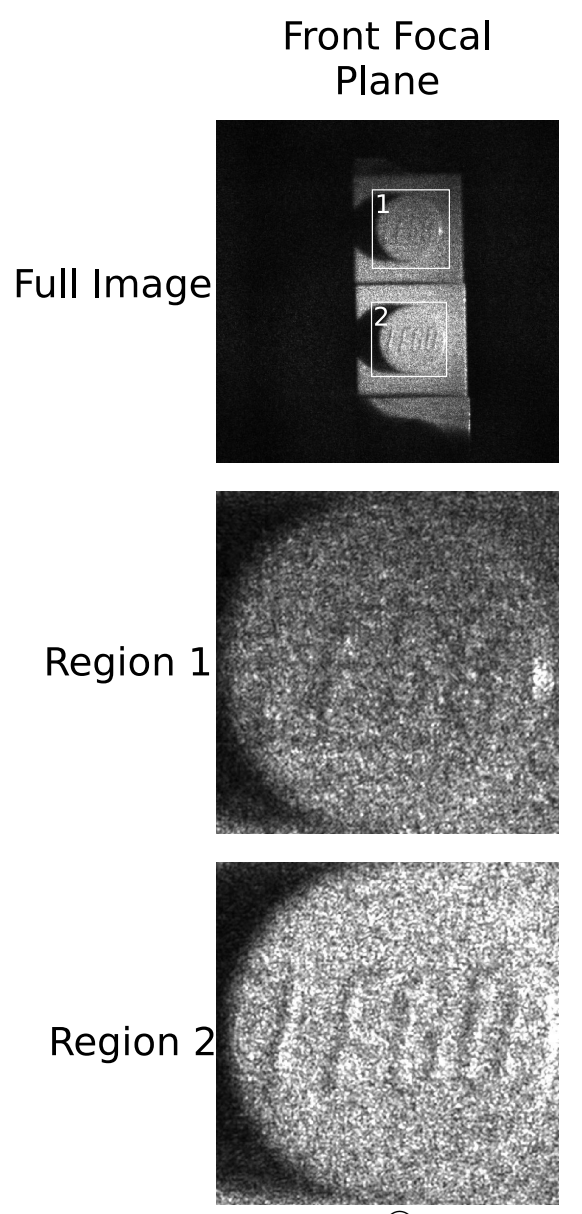

Figure 8. LEGO ${ }^{\circledR}$ block object DH, reconstructions and the overlapping approach EFIs.

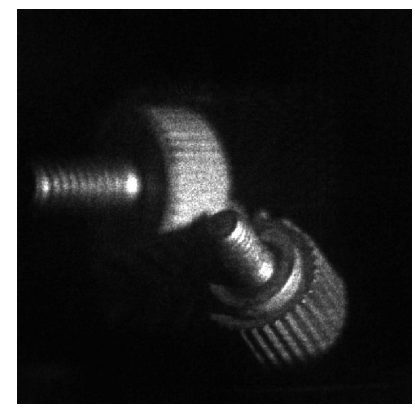

(a)

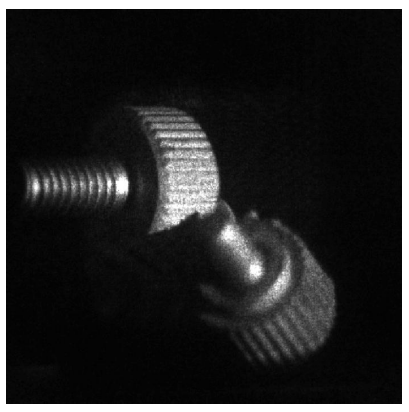

(b)

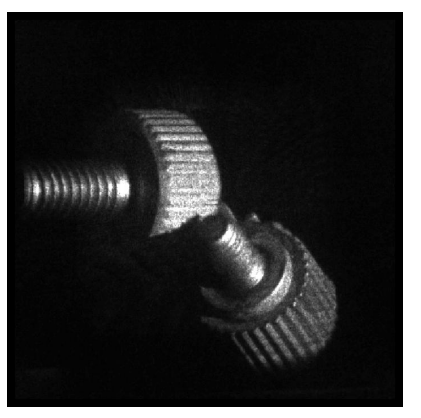

(c)

Figure 9. Bolts object DH: (a) front focal plane reconstruction, (b) back focal plane reconstruction and (c) EFI .

out of volumes of digital holographic reconstructions. Using DHs of real-world three-dimensional macroscopic objects we have experimentally verified our technique. Multiple approaches for creating EFIs have been described along with their disadvantages and advantages. The non-overlapping DFF algorithm is relatively fast but there is a significant loss of resolution. Our overlapping DFF algorithm is computationally expensive but has the advantages of high resolution. Objects can be illuminated with a speckle pattern to increase the accuracy of depth estimation, ${ }^{50}$ specifically in the case of objects with little or no texture. We intend to investigate the impact of a real physical background behind the objects. We have successfully created EFIs for scenes containing multiple and single objects and containing low and high contrast objects and have demonstrated an increase to 


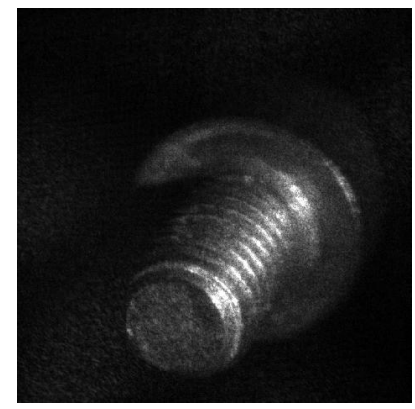

(a)

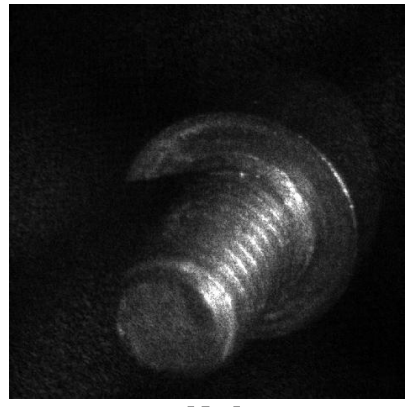

(b)

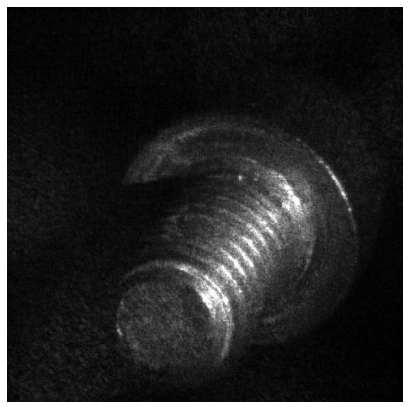

(c)

Figure 10. Bolt object DH: (a) front focal plane reconstruction, (b) back focal plane reconstruction and (c) $\mathrm{EFI}_{\mathrm{P}}$.

the depth of focus of our system from $0.8 \mathrm{~mm}$ to $20 \mathrm{~mm}$. To our knowledge, this is the first time that EFIs have been created for DHs containing macroscopic objects.

\section{Acknowledgements}

This publication has emanated from research conducted with the financial support of Science Foundation Ireland,

Enterprise Ireland, and and the Embark Initiative of the Irish Research Council for Science, Engineering, and Technology, and the European Commission through a Marie Curie Fellowship. LEGO ${ }^{\circledR}$ is a trademark of the LEGO Group of companies. LEGO ${ }^{\circledR}$ did not sponsor, authorize, or endorse this research.

\section{REFERENCES}

[1] Kreis, T., [Handbook of Holographic Interferometry], Wiley (2005).

[2] Onural, L. and Scott, P., "Digital decoding of in-line holograms," Optical Engineering 26, 1124-1132 (1987).

[3] Schnars, U. and Jüptner, W., [Digital Holography: Digital Hologram Recording, Numerical Reconstruction, and Related Techniques], Springer, Berlin (2004).

[4] Yamaguchi, I. and Zhang, T., "Phase-shifting digital holography," Applied Optics 22, 1268-1270 (1997).

[5] Naughton, T., Frauel, Y., Javidi, B., and Tajahuerce, E., "Compression of digital holograms for threedimensional object reconstruction and recognition," Applied Optics 41, 4124-4132 (2002).

[6] Maycock, J., McElhinney, C., Hennelly, B., Naughton, T., McDonald, J., and Javidi, B., "Reconstruction of partially occluded objects encoded in three-dimensional scenes by using digital holograms," Applied Optics 45, 2975-2985 (2006).

[7] Frauel, Y., Naughton, T., Matoba, O., Tajahuerce, E., and Javidi, B., "Three-dimensional imaging and processing using computational holographic imaging," Proceedings of the IEEE 94, 636-653 (2006).

[8] Yaroslavskii, L. and Merzlyakov, N., [Methods of Digital Holography], Consultants Bureau, New York (1980). Translated from Russian by Dave Parsons.

[9] Kim, D. and Javidi, B., "Distortion-tolerant 3-d object recognition by using single exposure on-axis digital holography," Optics Express 12, 5539-5548 (2004).

[10] Javidi, B. and Kim, D., "Three-dimensional-object recognition by use of single-exposure on-axis digital holography," Optics Letters 30, 236-238 (2005).

[11] Javidi, B., Moon, I., Yeom, S., and Carapezza, E., "Three-dimensional imaging and recognition of microorganism using single-exposure on-line (seol) digital holography," Optics Express 13, 4492-4506 (2005).

[12] Naughton, T., McDonald, J., and Javidi, B., "Efficient compression of Fresnel fields for Internet transmission of three-dimensional images," Applied Optics 42, 4758-4764 (2003).

[13] McElhinney, C., Shortt, A., Naughton, T., and Javidi, B., "Blockwise discrete fourier transform analysis of digital hologram data of three-dimensional objects," Proc. of Optics and Photonics, Colorado , 62-69 (2004).

[14] Yamaguchi, I., Yamamoto, K., Mills, G., and Yokota, M., "Image reconstruction only by phase data in phase-shifting digital holography," Applied Optics 45, 975-983 (2006). 
[15] Shortt, A., Naughton, T., and Javidi, B., "Compression of digital holograms of three-dimensional objects using wavelets," Optics Express 14, 2625-2630 (2006).

[16] Darakis, E. and Soraghan, J., "Reconstruction domain compression of phase-shifting digital holograms," Applied Optics 46, 351-356 (2007).

[17] Bertaux, N., Frauel, Y., Réfrégier, P., and Javidi, B., "Speckle removal using a maximum-likelihood technique with isoline gray-level regularization," Optics Society of America 21, 2283-2291 (2004).

[18] Maycock, J., Hennelly, B. M., McDonald, J. B., Naughton, T. J., Frauel, Y., Castro, A., and Javidi, B., "Reduction of speckle in digital holography by discrete fourier filtering," Journal of the Optical Society of America A 24, 2975-2985 (2007).

[19] Poon, T.-C., "Recent progress in optical scanning holography," Journal of Holography and Speckle 1, 6-25 (2004).

[20] Cuche, E., Marquet, P., and Depeursinge, C., "Spatial filtering for zero-order and twin-image elimination in digital off-axis holography," Applied Optics 39, 4070-4075 (2000).

[21] Takari, Y., Kawai, H., and Ohzu, H., "Hybrid holographic microscopy free of conjugate and zero-order images," Applied Optics 38, 4990-4996 (1999).

[22] Poon, T.-C., Kim, T., Indebetouw, G., Schilling, B., Wu, M., Shinoda, K., and Suzuki, Y., "Twin-image elimination experiments for three-dimensional images in optical scanning holography," Optics Letters 25, 215-217 (2000).

[23] Zhang, T. and Yamaguchi, I., "Three-dimensional microscopy with phase-shifting digital holography," Optics Letters 23, 1221-1223 (1998).

[24] Xu, L., Peng, X., Miao, J., and Asundi, A., "Studies of digital microscopic holography with applications to microstructure testing," Applied Optics 40, 5046-5051 (2001).

[25] Cuche, E., Marquet, P., and Depeursinge, C., "Simultaneous amplitude-contrast and quantitative phasecontrast microscopy by numerical reconstruction of fresnel off-axis holograms," Applied Optics 38, 6994-7001 (1999).

[26] Ferraro, P., Coppola, G., Nicola, S., Finizio, A., and Peirattini, G., "Digital holographic microscope with automatic focus tracking by detecting sample displacement in real time," Optics Letters 28, 1257-1259 (2003).

[27] Pedrini, G., Froning, P., Tiziani, H., and Santoyo, F., "Shape measurement of microscopic structures using digital holograms," Optics Communications 164 (1999).

[28] Mann, C., Yu, L., Lo, C., and Kim, M., "High-resolution quantitative phase-contrast microscopy by digital holography," Optics Express 13, 8693-8698 (2005).

[29] Ferraro, P., Grilli, S., Alfieri, D., Nicola, S., Finizio, A., Pierattini, G., Javidi, B., Coppola, G., and Striano, V., "Extended focused image in microscopy by digital holography," Optics Express 13, 6738-6749 (2005).

[30] Gustafsson, M. and Sebesta, M., "Refractometry of microscopic objects with digital holography," Applied Optics 43, 4796-4801 (2004).

[31] DaneshPanah, M. and Javidi, B., "Segmentation of 3d holographic images using bivariate jointly distributed region snake," Optics Express 14, 5143-5153 (2006).

[32] McElhinney, C., McDonald, J., Y. Frauel, A. Castro, B. J., and Naughton, T., "Depth-independent segmentation of three-dimensional objects encoded in single perspectives of digital holograms," Optics Letters $\mathbf{3 2}$, 1229-1231 (2007).

[33] Javidi, B. and Tajahuerce, E., "Three-dimensional object recognition by use of digital holography," Optics Letters 25, 610-612 (2000).

[34] Frauel, Y. and Javidi, B., "Neural network for three-dimensional object recognition based on digital holography," Optics Letters 26, 1478-1480 (2001).

[35] Javidi, B., Yeom, S., Moon, I., and Daneshpanah, M., "Real-time automated 3d sensing, detection and recognition of dynamic biological micro-organic events," Optics Express 14, 3806-3829 (2006).

[36] Pieper, R. and Korpel, A., "Image processing for extended depth of field," Applied Optics 22, 1449-1453 (1983).

[37] Ma, L., Wang, H., Li, Y., and Jin, H., "Numerical reconstruction of digital holograms for three-dimensional shape measurement," Journal of Optics A: Pure and Applied Optics 6, 396- (2004). 
[38] Xu, L., Miao, J., and Asundi, A., "Properties of digital holography based on in-line configuration," Optical Engineering 39, 3214-3219 (2000).

[39] Bruning, J., Herriott, D., Gallagher, J., Rosenfeld, D., White, A., and Brangaccio, D., "Digital wavefront measuring interferometer for testing optical surfaces and lenses," Applied Optics 13, 2693-2703 (1974).

[40] Goodman, J., [Introduction to Fourier Optics], Roberts and Company, Englewood, Colorado, third ed. (2005).

[41] Caulfield, H., [Handbook of Optical Holography], Academic Press, New York (1979).

[42] Gillespie, J. and King, R., "The use of self-entropy as a focus measure in digital holography," Pattern Recognition Letters 9, 19-25 (1989).

[43] Liebling, M. and Unser, M., "Autofocus for digital fresnel holograms by use of a fresnelet-sparsity criterion," Journal of the Optical Society of America A 21, 2424-2430 (2004).

[44] Dubois, F., Schockaert, C., Callens, N., and Yourassowsky, C., "Focus plane detection criteria in digital holography microscopy by amplitude analysis," Optics Express 14, 5895-5908 (2006).

[45] Malkiel, E., Abras, J., and Katz, J., "Automated scanning and measurement of particle distributions within a holographic reconstructed volume," Measurement of Science and Technology 15, 601-612 (2004).

[46] Burns, N. and Watson, J., "Data extraction from underwater holograms of marine organisms," Proceedings of IEEE Oceans 07 (IEEE, 2007) p. 1. .

[47] Subbarao, M., Choi, T., and Nikzad, N., "Focusing techniques," Optical Engineering 32, 2824-2836 (1993).

[48] Fienup, J., "Invariant error metrics for image reconstruction," Applied Optics 36, 8352-8357 (1997).

[49] C.P. Mc Elhinney, B. H. and Naughton, T., "Extended focused imaging for digital holograms of macroscopic three-dimensional objects," Applied Optics 47, D71-D79 (2008).

[50] Frey, S., Thelen, A., Hirsch, S., and Hering, P., "Generation of digital textured surface models from hologram recordings," Applied Optics 46, 1986-1993 (2007). 